\title{
Tunable and wavelength interval precisely controlled erbium-doped fiber laser by employing the fused taper technology
}

\author{
Qi Zhao (赵 琦) ${ }^{1,2}$ ，Li Pei (裴 丽) $)^{1,2^{*}}$ ，Zuliang Ruan (阮祖亮) ${ }^{1,2}$ ， Jingjing Zheng (郑晶晶) $)^{1,2}$ ， Jianshuai Wang (王建帅) $)^{1,2}$ ， \\ Min Tang (汤 敏) $)^{1,2}$, Jing Li (李 晶) ${ }^{1,2}$, and Tigang Ning (宁提纲) $)^{1,2}$ \\ ${ }^{1}$ Institute of Lightwave Technology, Beijing Jiaotong University, Beijing 100044, China \\ ${ }^{2}$ Key Laboratory of All Optical Network and Advanced Telecommunication Network of Ministry of Education, Beijing Jiaotong University, Beijing 100044, China
}

*Corresponding author: lipei@bjtu.edu.cn

Received May 31, 2021 | Accepted July 16, 2021 | Posted Online September 27, 2021

\begin{abstract}
A tunable multi-wavelength erbium-doped fiber laser with precise wavelength interval control is reported theoretically and experimentally in this paper. It is made up of a Mach-Zehnder interferometer (MZI) filter and a Sagnac filter and supplemented by the four-wave-mixing effect. Compared with other filters, the proposed MZI filter based on the fused taper technology can change the wavelength interval more flexibly. The experiment result shows that wavelength tuning can be achieved, and the tuning range can reach $\sim 15 \mathrm{~nm}$. Moreover, the variation in the number of wavelengths is also realized. The maximum side-mode suppression ratio can reach $39 \mathrm{~dB}$.
\end{abstract}

Keywords: erbium-doped fiber laser; fused taper technology; wavelength interval.

DOI: 10.3788/COL202220.011402

\section{Introduction}

In recent years, the multi-wavelength fiber laser has been practically used in many fields due to its excellent performance ${ }^{[1-3]}$. In order to adapt to the requirements of different environments, the output of the laser needs to be more flexible. Thus, the output characteristics of multi-wavelength fiber lasers, such as wavelength number, wavelength output, and wavelength interval of multi-wavelength fiber lasers, are worth being investigated ${ }^{[4]}$. Based on the above description, researchers have tried many methods ${ }^{[5,6]}$.

Tunable fiber lasers can usually be implemented by using comb filters based on the principle of fast and slow axis interference, such as Sagnac filter and Lyot filter. In 2004, Song et al. used a Sagnac filter to make a self-seeded Brillouin scattering laser. The tuning range of the laser can be $14.5 \mathrm{~nm}^{[7]}$. After that, Gao et al. extended the tuning range of the output wavelength to $37 \mathrm{~nm}^{[8]}$. But, the flexibility of the output wavelength is not enough. Considering the discrete wavelength selectivity of the fiber Bragg grating (FBG), Liu et al. cascaded mismatching long-period fiber gratings (LFPGs) and comb filters to obtain switchable and tunable wavelength output ${ }^{[9]}$. However, with the increase in the number of FBGs, the insertion loss and complexity of the laser system are getting higher, and the FBGs are easily damaged in applications. In 2017, Li et al. demonstrated an erbium-doped fiber laser (EDFL), which is composed of a
Lyot filter and a nonlinear optical loop mirror (NOLM) ${ }^{[4]}$. The output of the laser has both switchability and tunability. The output wavelength is up to 12 wavelengths, but the tuning range is only $8 \mathrm{~nm}$. Zheng et al. cascaded the self-made filter with a Sagnac loop to obtain switchable and tunable output ${ }^{[10]}$. However, the change of the wavelength interval in the laser structure mentioned above is either difficult to achieve or has great randomness. In the past, many methods have been proposed to realize the change of wavelength interval. Wang et al. proposed a novel switchable multi-wavelength fiber laser based on a Lyot-Sagnac comb filter, and two fixed wavelength intervals were obtained ${ }^{[11]}$. Then, a three wavelength intervals tunable multi-wavelength fiber laser was proofed by Wang et al. ${ }^{[12]}$. The filter used in the laser is composed of two sections of polarization maintaining fiber (PMF). In addition, researchers have also used parallel dual Lyot filters ${ }^{[13]}$, polarizing beam splitter (PBS)-based two-stage cascaded Mach-Zehnder interferometer $(\mathrm{MZI})^{[14]}$, optical variable delay line (OVDL) $)^{[15,16]}$, etc. to obtain wavelength output with adjustable intervals. However, it should be noted that most of the structures cannot achieve wavelength tuning and wavelength interval adjustment at the same time, as well as the change of wavelength number.

In this paper, a tunable multi-wavelength EDFL with precise wavelength interval control is proposed. It is proved theoretically and experimentally that the comb filter has a controllable 
wavelength interval by using taper technology on one arm of the MZI. By inserting the proposed MZI filter into the EDFL, the stable laser outputs with the wavelength intervals of $1 \mathrm{~nm}, 0.7 \mathrm{~nm}$, and $0.5 \mathrm{~nm}$ are obtained in the experiments. Furthermore, the change in the number of output lasers and wavelength tuning can also be realized. The side-mode suppression ratios (SMSRs) of the outputs are larger than $35 \mathrm{~dB}$. Compared with other methods, the output characteristics of the structure, such as the tunability of wavelength interval and the switchability of wavelength number, have been greatly improved.

\section{Experimental Setup and Principle}

The schematic of the proposed fiber laser cavity is shown in Fig. 1. The 7-m-long erbium-doped fiber (EDF) is used as the gain medium. The model of the fiber is Nufern, EDFC-980$\mathrm{HP}$, and the maximum absorption coefficient is $6 \mathrm{~dB} / \mathrm{m}^{[17]}$. The $3 \mathrm{~km}$ SMF is used to introduce the four-wave mixing (FWM). It can reduce the wavelength competition, thereby the number of outputs increase ${ }^{[18]}$. Optical coupler $1\left(\mathrm{OC}_{1}\right)$, polarization controller $\left(\mathrm{PC}_{1}\right)$, and $0.3 \mathrm{~m}$ PMF form the Sagnac loop located on the left side of the laser. The right part is the proposed MZI filter, which is theoretically analyzed and simulated as follows. $\mathrm{OC}_{2}$ and $\mathrm{PC}_{2}$ are utilized to compose the resonant cavity of the laser. One port of $\mathrm{OC}_{2}$ is employed to measure the characteristics of the output wavelengths. Besides, the spectral analyzer applied in the experiment has a resolution of $0.05 \mathrm{~nm}$.

\subsection{Characteristics of the MZI filter}

Figure 2 is the schematic diagram of the proposed MZI filter used in the experiment. Different from the common MZI filters, one arm of the proposed filter is composed of a tapered fiber obtained by tapering. The transfer functions of the filter at

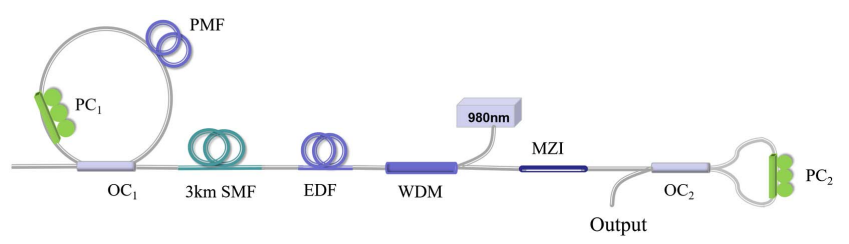

Fig. 1. Schematic diagram of the tunable and wavelength interval controlled EDFL using the proposed MZI filter.

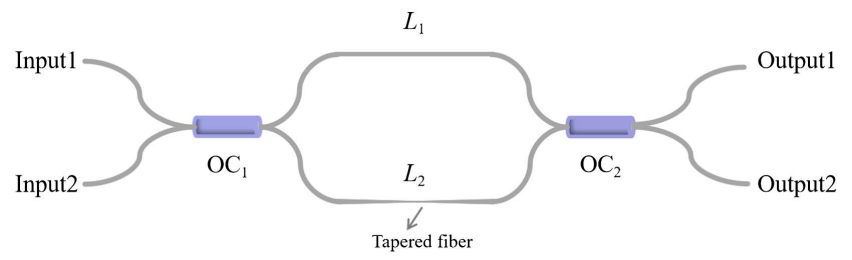

Fig. 2. Schematic diagram of the proposed MZI filter composed of tapered fiber. output 1 and output 2 are shown in Eq. $(1)^{[5]}$. Among them, $\lambda$ is the working wavelength, and the length difference between the two optical paths in MZI filter is expressed by $\Delta L . n$ is the effective refractive index of the SMF, which can be set as 1.45 according to the actual situation. Moreover, $\varphi$ represents the linear phase shift. Because of the choice of parameters in simulation and experiment, the influence of nonlinear phase shift can be ignored. Therefore, Eq. (1) is only an expression for linear phase shift. According to Eq. (2), the adjacent interval of the output wavelength can be calculated and obtained. Figure 3 shows the transmission characteristics of the filter obtained in the simulation, where the wavelength intervals are $1 \mathrm{~nm}, 0.7 \mathrm{~nm}, 0.5 \mathrm{~nm}$, and $0.2 \mathrm{~nm}$. The corresponding $\Delta L$ between the two arms is $1656 \mu \mathrm{m}, 2367 \mu \mathrm{m}, 3313 \mu \mathrm{m}$, and $8284 \mu \mathrm{m}$, respectively.

$$
\begin{gathered}
T=\frac{1}{2}(1-\cos \varphi), \quad \varphi=\frac{2 \pi n}{\lambda} \Delta L, \\
\Delta \lambda=\frac{\lambda^{2}}{n \Delta L} .
\end{gathered}
$$

Figure 4 shows the taper platform required to realize the wavelength interval adjustment of the MZI transmission

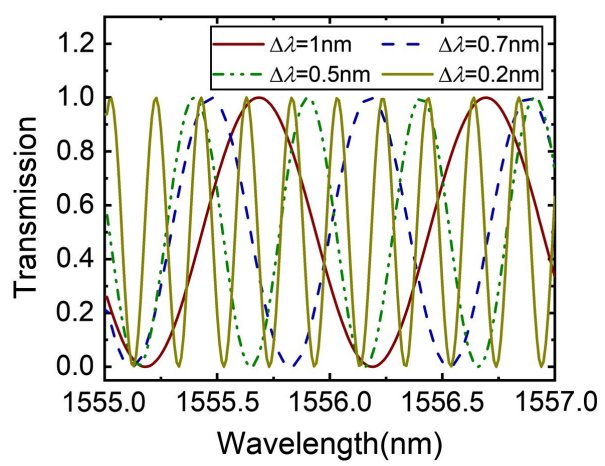

Fig. 3. Simulation of the transmission spectra of the MZI filter when the wavelength intervals are $1 \mathrm{~nm}, 0.7 \mathrm{~nm}, 0.5 \mathrm{~nm}$, and $0.2 \mathrm{~nm}$.

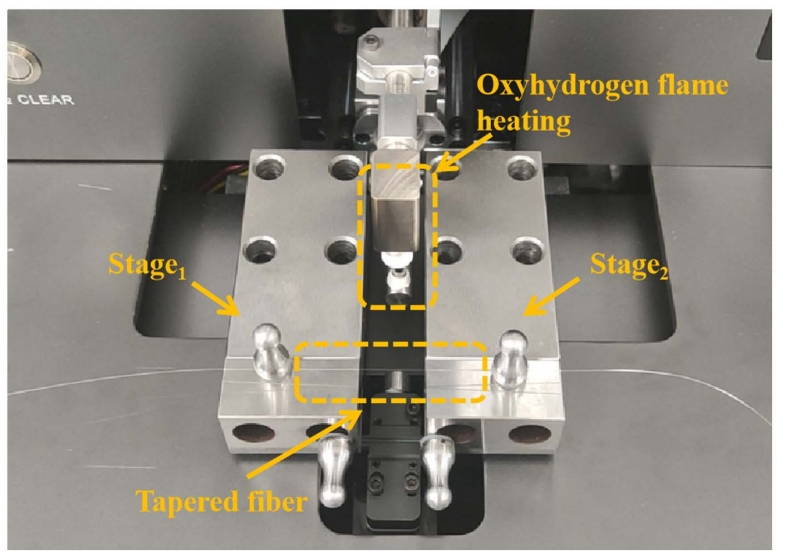

Fig. 4. Camera image of the taper platform and the taper fiber. 

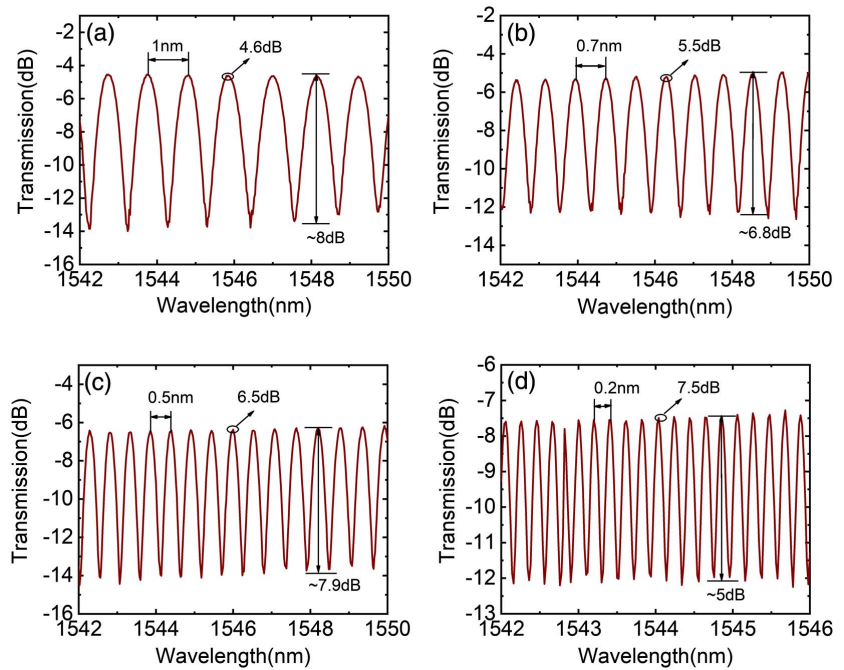

Fig. 5. Measurement of transmission of the MZI filter when the wavelength interval is (a) $1 \mathrm{~nm}$, (b) $0.7 \mathrm{~nm}$, (c) $0.5 \mathrm{~nm}$, and (d) $0.2 \mathrm{~nm}$.

spectrum. One arm of the MZI filter is fixed on two translation stages ( Stage $_{1}$ and Stage ${ }_{2}$ ), which is heated and stretched by oxyhydrogen flame. During the stretching process, the two translation stages move outward, and the moving range can be set according to the calculation results ${ }^{[19]}$. This method greatly improves the flexibility of adjusting the wavelength interval. In theory, any value of the wavelength interval can be obtained.

In order to verify the reliability of the simulation, one arm of the MZI filter is tapered according to the calculation results, and the tapered length is consistent with the $\Delta L$ in the simulation. The SMF used in the filter is G.652. The distance between the two stages of the taper platform is $21 \mathrm{~mm}$, which means that the 21-mm-long SMF is tapered. The diameter of the SMF gradually changed to $8.66 \mu \mathrm{m}, 8.4 \mu \mathrm{m}, 8.36 \mu \mathrm{m}$, and $7.6 \mu \mathrm{m}$ with the increase of taper length. The fiber is easy to break when the two stages are too close, and the taper will produce a large error when the distance is too large. The transmission of the MZI filter is shown in Fig. 5. The wavelength intervals are $1 \mathrm{~nm}, 0.7 \mathrm{~nm}$, $0.5 \mathrm{~nm}$, and $0.2 \mathrm{~nm}$, respectively, which are the same as the simulation results. With the increase of the taper length, the loss of the filter increases from $4.6 \mathrm{~dB}$ to $7.5 \mathrm{~dB}$.

\subsection{Principle of the cascaded filter}

When the wavelength interval is determined, the Sagnac filter starts to select the output wavelength because of its large envelope, so that wavelength tuning and number switching can be realized. The principle of the cascading MZI filter and Sagnac filter is shown in Fig. 6, where Gain Profile (0) and Gain Profile (1) are the spectra of the Sagnac filter. Gain Profile (2) is the spectrum of the MZI filter with wavelength interval of $0.5 \mathrm{~nm}$. According to the principle of the laser, when the gain in the cavity is greater than the loss, laser output is produced. By adjusting the PCs in the Sagnac filter to tune the output profile, if Gain Profile (0) appears, lasings at $\lambda_{1}, \lambda_{2}$, and $\lambda_{3}$ are generated. Continuing to adjust the PC, the output spectrum

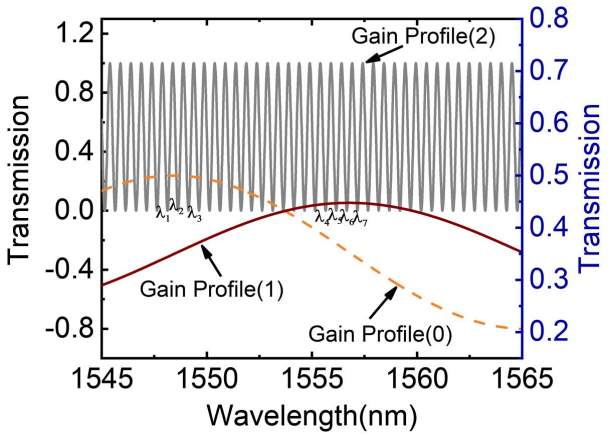

Fig. 6. Principle of the cascading MZI filter and Sagnac filter.

becomes Gain Profile (1), and $\lambda_{4}, \lambda_{5}, \lambda_{6}$, and $\lambda_{7}$ are emitted. On the other hand, when the gain profile is fixed, wavelength switching can be achieved by adjusting $\mathrm{PC}_{2}$. As shown in Fig. 6, it is assumed that the initial gains at $\lambda_{1}, \lambda_{2}$, and $\lambda_{3}$ are large enough to output the laser. However, adjusting $\mathrm{PC}_{2}$ will change the polarization hole burning effect (PHB) in the laser cavity, which may change the loss and gain of certain wavelengths. When the loss is greater than the gain, the lasers cannot be output, which will eventually lead to a change in the number of output wavelengths ${ }^{[17,20,21]}$.

\section{Experimental Results and Discussions}

In the experiment, the two arms of the MZI filter are with the same length initially. Then, one of the arms is tapered by the tapered platform according to the set length. It can be seen from Section 2 that the wavelength interval of the outputs is determined by the taper length. When the pump power is $200 \mathrm{~mW}$, the cascaded filter selects the wavelength and closely cooperates with the FWM, and a variety of lasing states can be generated. Figure 7 shows the spectra of the output wavelengths, in which
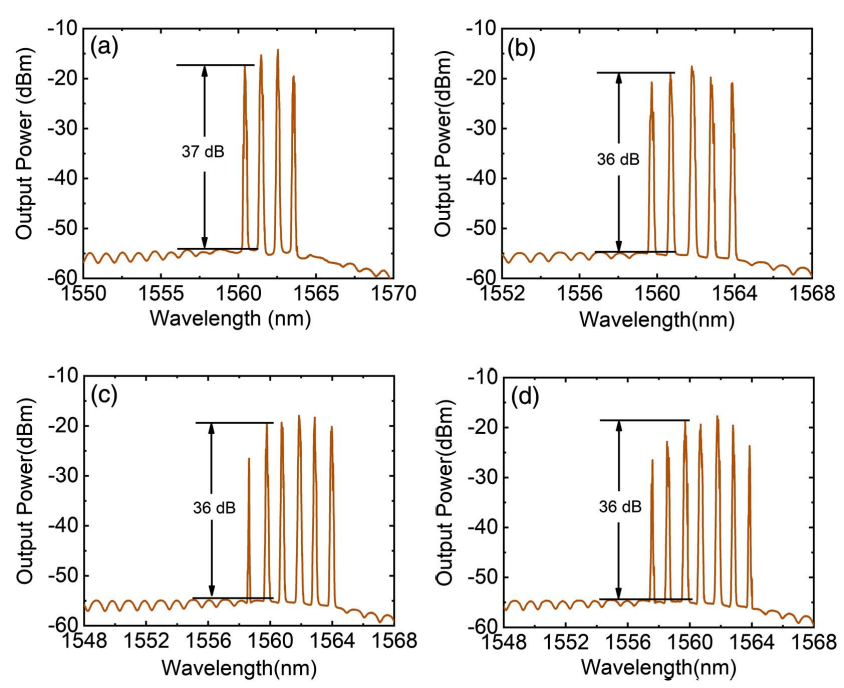

Fig. 7. Spectra of the output wavelengths when the wavelength interval is $1 \mathrm{~nm}$ : (a) quadruple, (b) quintuple, (c) sextuple, and (d) septuple wavelengths. 
quadruple, quintuple, sextuple, and septuple wavelengths can be obtained by adjusting $\mathrm{PC}_{2}$. Since the taper length is set to $1656 \mu \mathrm{m}$, the wavelength interval is $1 \mathrm{~nm}$.

Due to the existence of the Sagnac filter, the output wavelength can be tuned in a certain wavelength range when $\mathrm{PC}_{1}$ is adjusted properly. Figures $8(\mathrm{a}), 8(\mathrm{~b})$, and $8(\mathrm{c})$ are the tunable spectra of the quadruple, quintuple, and sextuple wavelengths with a tuning step of $\sim 1 \mathrm{~nm}$. The maximum tuning range is $\sim 15 \mathrm{~nm}$. Theoretically, there should be wavelength outputs for each step in the tuning range. However, due to the characteristics of the amplified spontaneous emission (ASE) spectrum of the EDF, PHB, laser cavity loss, etc., the experimental results cannot be completely the same as the theory. At the same time, it is found that the tuning range decreases with the increase of the number of output wavelengths. If the gain range and gain
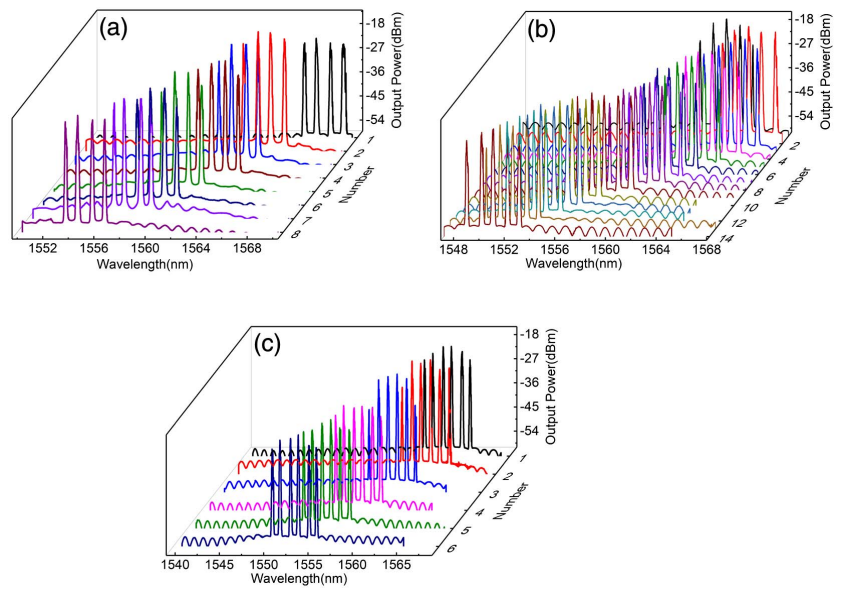

Fig. 8. Tunable output spectra of the proposed EDFL: (a) quadruple, (b) quintuple, and (c) sextuple wavelengths.
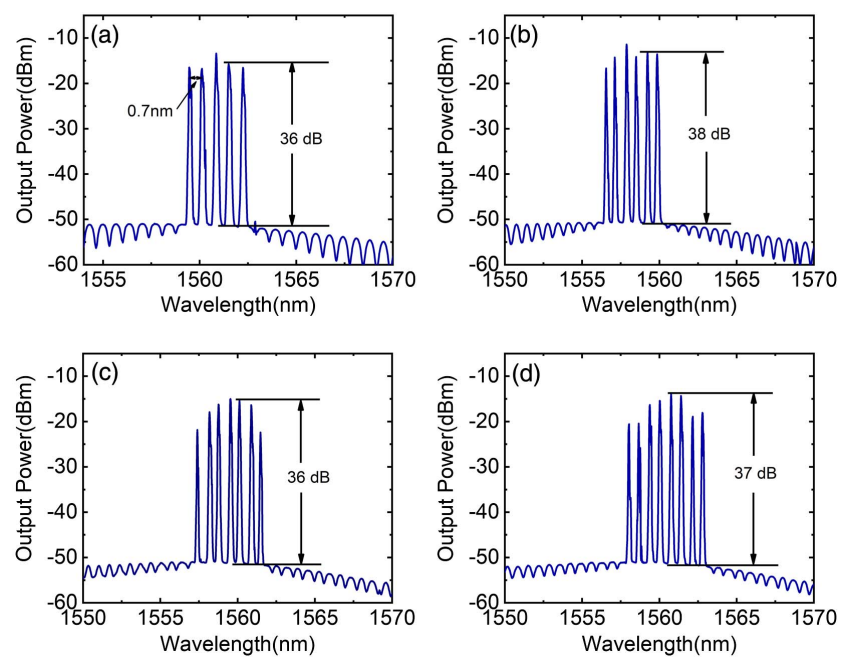

Fig. 9. Spectra of the output wavelengths when the wavelength interval is $0.7 \mathrm{~nm}$ : (a) quintuple, (b) sextuple, (c) septuple, and (d) octuple wavelengths. coefficient of the EDF are expanded, the tuning range of the wavelength will be increased.

By slowly moving Stage ${ }_{1}$ and Stage ${ }_{2}$, the length difference $(\Delta L)$ between the two optical paths of the MZI is increased to $2367 \mu \mathrm{m}$, and the wavelength interval becomes $0.7 \mathrm{~nm}$, which are shown in Fig. 9. The figure shows the spectra of quintuple, sextuple, septuple, and octuple wavelengths. When the tapering continues, $\Delta L$ becomes $3313 \mu \mathrm{m}$, and the output wavelengths are shown in Fig. 10 with the wavelength interval of $0.5 \mathrm{~nm}$. The maximum number of wavelengths is eight. The maximum SMSR is $39 \mathrm{~dB}$. Considering that if the length of the taper is too long, the fiber will be too thin and easily damaged, so the wavelength interval is not further reduced. Nevertheless, the mentioned wavelength intervals have been suitable for application in wavelength division multiplexing (WDM) systems. Wavelength tuning can also be achieved when the wavelength intervals are $0.7 \mathrm{~nm}$ and $0.5 \mathrm{~nm}$. However, the randomness of the output is greatly increased. If the polarization states in the laser cavity can be better controlled, the results should be improved.

The stability of the proposed tunable multi-wavelength EDFL is verified by recording the output spectra every $5 \mathrm{~min}$.
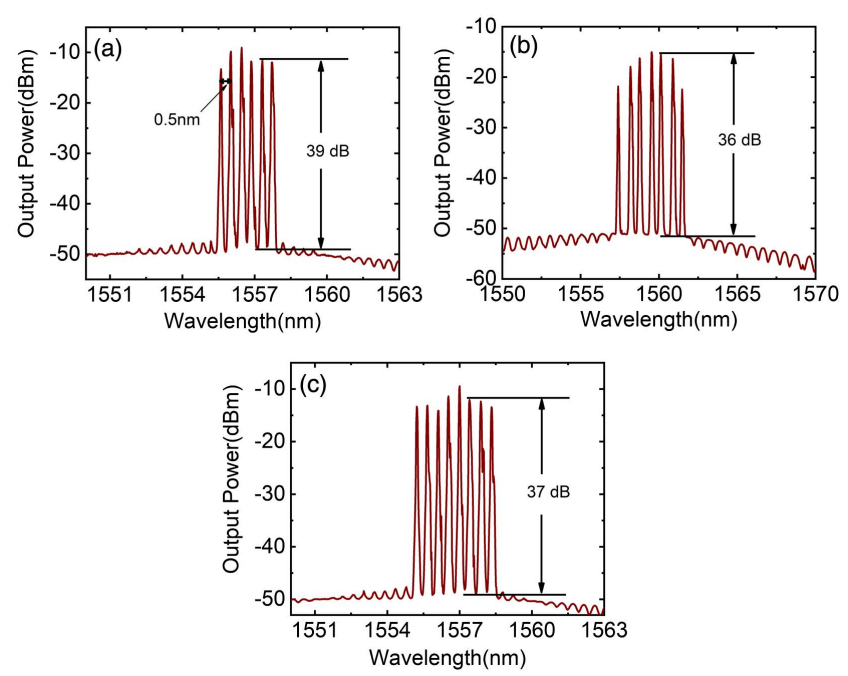

Fig. 10. Spectra of the output wavelengths when the wavelength interval is $0.5 \mathrm{~nm}$ : (a) sextuple, (b) septuple, and (c) octuple wavelengths.
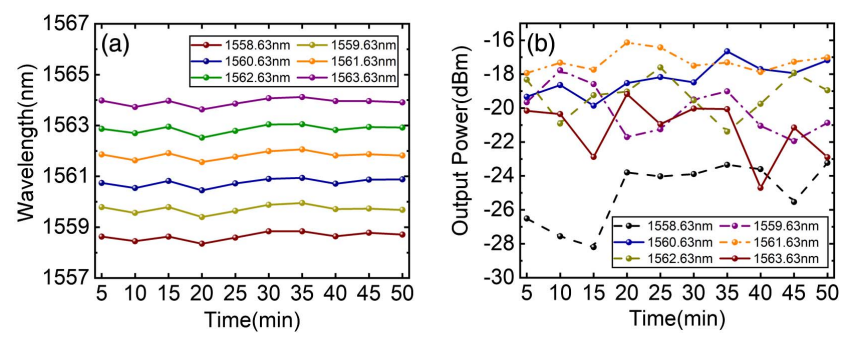

Fig. 11. (a) Wavelength drift and (b) power fluctuation for each wavelength within $50 \mathrm{~min}$. 
Figures 11(a) and 11(b) are the wavelength outputs and power measurements of sextuple wavelengths with a wavelength interval of $1 \mathrm{~nm}$ in $50 \mathrm{~min}$. It can be seen from the figure that the maximum wavelength shift and power fluctuation are less than $0.25 \mathrm{~nm}$ and $2.5 \mathrm{~dB}$, respectively. The experimental results show that the proposed laser has good stability. The intense wavelength competition and external interference in the laser cavity make it difficult to maintain long-term stable output at septuple wavelengths and octuple wavelengths ${ }^{[9,13]}$. The stability can be improved by placing the EDFL on the anti-interference optical platform or using a highly nonlinear fiber instead of an SMF to reduce the cavity length ${ }^{[22,23]}$.

\section{Conclusions}

In summary, a tunable and wavelength interval precisely controlled multi-wavelength EDFL has been reported theoretically and experimentally in this paper. The EDFL is based on the MZI filter and Sagnac filter with the assistance of FWM. In this paper, the principle of the MZI filter is analyzed, and the output spectra are obtained by simulation. The output wavelengths of the laser can be achieved with multiple different wavelength intervals by using the tapering platform to change the length difference between the two optical paths in the MZI filter. The wavelength intervals of $1 \mathrm{~nm}, 0.7 \mathrm{~nm}$, and $0.5 \mathrm{~nm}$ are obtained in the experiments. Furthermore, by adjusting the polarization states of the PCs, the wavelength tuning range is up to $15 \mathrm{~nm}$, and the maximum number of output wavelengths can reach eight. The SMSRs of the outputs are larger than $35 \mathrm{~dB}$. The proposed tunable EDFL with precise wavelength interval control can be applied to different fields due to its flexible output characteristics.

\section{Acknowledgement}

This work was supported by the National Key R\&D Program of China (No. 2018YFB1801003) and in part by the National Natural Science Foundation of China (No. 61827817).

\section{References}

1. L. Huang, X. Song, P. Chang, W. Peng, W. Zhang, F. Gao, F. Bo, G. Zhang, and J. Xu, "All-fiber tunable laser based on an acousto-optic tunable filter and a tapered fiber," Opt. Express 24, 7449 (2016).

2. Z. Zhang, L. Zhan, and Y. Xia, "Tunable self-seeded multiwavelength Brillouin-erbium fiber laser with enhanced power efficiency," Opt. Express 15, 9731 (2007).

3. S. Pan, C. Lou, and Y. Gao, "Multiwavelength erbium-doped fiber laser based on inhomogeneous loss mechanism by use of a highly nonlinear fiber and a Fabry-Perot filter," Opt. Express 14, 1113 (2006).
4. Y. Li, J. Tian, M. Quan, and Y. Yao, "Tunable multiwavelength Er-doped fiber laser with a two-stage Lyot filter," IEEE Photon. Technol. Lett. 29, 287 (2017).

5. H. Han, X. Li, S. Zhang, and M. Han, "Precise wavelength control of Yb-doped fiber laser using fused tapered fiber technology," J. Lightwave Technol. 37, 715 (2019).

6. Q. Zhao, L. Pei, L. Wu, T. Ning, J. Zheng, and J. Li, "Wide tuning range and high OSNR self-seeded multi-wavelength Brillouin-erbium fiber laser based on a Lyot filter," Appl. Opt. 57, 10474 (2018).

7. Y. J. Song, L. Zhan, S. Hu, Q. H. Ye, and Y. X. Xia, "Tunable multiwavelength Brillouin-erbium fiber laser with a polarization-maintaining fiber Sagnac loop filter," IEEE Photon. Technol. Lett. 16, 2015 (2004).

8. W. Gao, M. Liao, T. Cheng, T. Suzuki, and Y. Ohishi, "Tunable Brillouinerbium comb fiber laser in a linear cavity with a single-mode tellurite fiber," IEEE Photon. Technol. Lett. 25, 51 (2013).

9. X. Liu, L. Zhan, S. Luo, Y. Wang, and Q. Shen, "Individually switchable and widely tunable multiwavelength erbium-doped fiber laser based on cascaded mismatching long-period fiber gratings," J. Lightwave Technol. 29, 3319 (2011).

10. J. Zheng, A. Yang, T. Wang, X. Zeng, N. Cao, M. Liu, F. Pang, and T. Wang, "Wavelength-switchable vortex beams based on a polarization-dependent microknot resonator," Photon. Res. 6, 396 (2018).

11. B. Wang, Z. Cao, F. Ai, B. Chen, S. Zhen, X. Shi, and B. Yu, "Switchable quadruple-wavelength erbium-doped fiber ring laser based on two-segment Lyot-Sagnac filter," Opt. Laser Technol. 42, 357 (2010).

12. W. Wang, H. Meng, X. Wu, W. Wang, H. Xue, C. Tan, and X. Huang, "Three channel-spacing switchable multiwavelength fiber laser with two segments of polarization-maintaining fiber," IEEE Photon. Technol. Lett. 24, 470 (2012).

13. Q. Zhao, L. Pei, J. Zheng, M. Tang, Y. Xie, J. Li, and T. Ning, "Switchable multi-wavelength erbium-doped fiber laser with adjustable wavelength interval," J. Lightwave Technol. 37, 3784 (2019).

14. Z.-C. Luo, A.-P. Luo, and W.-C. Xu, "Tunable and switchable all-fiber comb filter using a PBS-based two-stage cascaded Mach-Zehnder interferometer," Opt. Commun. 284, 4167 (2011).

15. D. Chen, H. Ou, H. Fu, S. Qin, and S. Gao, "Wavelength-spacing tunable multi-wavelength erbium-doped fiber laser incorporating a semiconductor optical amplifier," Laser Phys. Lett. 4, 287 (2006).

16. D. Chen, S. Qin, and S. He, "Channel-spacing-tunable multi-wavelength fiber ring laser with hybrid Raman and erbium-doped fiber gains," Opt. Express 15, 930 (2007).

17. Q. Zhao, L. Pei, J. Zheng, M. Tang, Y. Xie, J. Li, and T. Ning, "Switchable, widely tunable and interval-adjustable multi-wavelength erbium-doped fiber laser based on cascaded filters," J. Lightwave Technol. 38, 2428 (2020).

18. Q. Zhao, L. Pei, M. Tang, Y. Xie, Z. Ruan, J. Zheng, and T. Ning, "Switchable multi-wavelength erbium-doped fiber laser based on core-offset structure and four-wave-mixing effect," Opt. Fiber Technol. 54, 102111 (2020).

19. M. Han, X. Li, S. Zhang, H. Han, J. Liu, and Z. Yang, "Tunable and channel spacing precisely controlled comb filters based on the fused taper technology," Opt. Express 26, 265 (2018).

20. Q. Zhao, L. Pei, J. Zheng, M. Tang, Y. Xie, J. Li, and T. Ning, "Tunable and interval-adjustable multi-wavelength erbium-doped fiber laser based on cascaded filters with the assistance of NPR," Opt. Laser Technol. 131, 106387 (2020).

21. L. Zeng, X. Sun, Z. Chang, Y. Hu, and J. Duan, "Tunable phase-shifted fiber Bragg grating based on a microchannel fabricated by a femtosecond laser," Chin. Opt. Lett. 19, 030602 (2021).

22. M. Wang, M. Liu, Y. Chen, D. Ouyang, J. Zhao, J. Pei, and S. Ruan, "Stable noise-like pulse generation in all-PM mode-locked Tm-doped fiber laser based on NOLM," Chin. Opt. Lett. 19, 091402 (2021).

23. H. Chen, X. Jiang, S. Xu, and H. Zhang, "Recent progress in multi-wavelength fiber lasers: principles, status, and challenges," Chin. Opt. Lett. 18, 041405 (2020). 